\title{
Clinicoradiological characteristics, management and prognosis of primary myeloid sarcoma of the central nervous system: A report of four cases
}

\author{
BAO YANG ${ }^{1 *}$, CHENLONG YANG ${ }^{2 *}$, JINGYI FANG $^{2}$, JUN YANG $^{1}$ and YULUN XU ${ }^{1}$ \\ ${ }^{1}$ Department of Neurosurgery, China National Clinical Research Center for Neurological Diseases, Beijing Tiantan Hospital, \\ Capital Medical University Dongcheng, Beijing 100050; ${ }^{2}$ Department of Neuropathology, \\ Beijing Neurosurgical Institute, Capital Medical University, Dongcheng, Beijing 100050, P.R. China
}

Received February 5, 2016; Accepted December 9, 2016

DOI 10.3892/ol.2017.6620

\begin{abstract}
Myeloid sarcoma (MS) is a localized tumor composed of premature precursors of granulocytic cells, which may occur in any organ and most commonly involves the soft tissue and musculoskeletal system. This malignancy may occur in the presence or absence of hematological disorders. Primary MS involving the central nervous system (CNS-MS) is rare, and has only been described in a small number of isolated case reports. The diagnosis of CNS-MS is challenging and strategies for its management are undefined. The present study describes 4 cases of CNS-MS. The hematological indices at admission were normal and all patients presented with location-associated nonspecific symptoms. In magnetic resonance imaging scans, the tumors appeared isointense on T1-weighted and T2-weighted images, with marked enhancement following contrast agent administration. Only 1 patient progressed to acute myeloblastic leukemia (AML), with this occurring 3 weeks following histological diagnosis. During the follow-up period, the patient with AML succumbed to the disease, local recurrence was noted in another patient for which a second surgery was requested and no progression was observed in the remaining 2 patients. CNS-MS has unique radiological characteristics and, due to its diffuse tissue infiltration, gross total resection is challenging. It is important for clinicians to be aware of potential hematological disorders in patients with CNS-MS.
\end{abstract}

Correspondence to: Professor Yulun $\mathrm{Xu}$, Department of Neurosurgery, China National Clinical Research Center for Neurological Diseases, Beijing Tiantan Hospital Affiliated to Capital Medical University, 6 Tiantan Xili, Dongcheng, Beijing 100050, P.R. China

E-mail: xuhuxi@sina.cn

${ }^{*}$ Contributed equally

Key words: myeloid sarcoma, central nervous system, leukemia, brain tumor, spinal tumor
A combined surgical and chemotherapeutic strategy may be able to provide long-term control of this malignancy.

\section{Introduction}

Myeloid sarcoma (MS) is a rare solid neoplasm composed of primitive myeloid cells, which may occur in any organ and most frequently involves the soft tissue and musculoskeletal system. MS is most commonly detected in association with acute myeloblastic leukemia (AML), myelodysplastic syndrome or myeloproliferative disorders and typically occurs during the course of active leukemia or following remission (1-4). However, MS may occasionally occur de novo without any evidence of concomitant hematological disease $(5,6)$. MS most frequently affects the bone, periosteum, lymph nodes and skin, but unusual presentations of MS in the breast, ovary, rectum, pancreas and urinary bladder have also been reported in previous studies (7-9). Central nervous system involvement of MS (CNS-MS) is very rare, with a reported incidence of $3.25 \%$ in patients with MS (10). The presence of this tumor without hematological disorders has been described in a small number of isolated case reports $(5,6,11)$.

The present study describes 4 cases of primary CNS-MS, in which the results of hematological examinations during hospitalization were all normal. To the best of our knowledge, this is the largest number of cases of this disease reported in a single study. Radiological findings, histopathological characteristics, clinical presentation and disease management were analyzed in the present study.

\section{Materials and methods}

The present study was approved by the Ethics Board of Capital Medical University (Beijing, China). Informed consent was obtained from the patients or their guardians in the case of the patient being $<18$ or unable to make informed medical decisions. The study included 3 males and 1 female who were admitted to Beijing Tiantan Hospital (Beijing, China) between January 2013 and December 2015, with a mean age of 28.5 years (range, 6-54). 
All patients underwent surgical treatment, and CNS-MS diagnosis was based on pathological and immunohistochemical staining. The resected specimens were fixed in $10 \%$ buffered formalin and embedded in paraffin. The slides were dried at $60^{\circ} \mathrm{C}$ for $1 \mathrm{~h}$ followed by deparaffinization in xylene (slides were immersed in xylene for $10 \mathrm{~min}$ and subsequently immersed in fresh xylene for $10 \mathrm{~min}$ ), rehydration using graded ethanol $(100 \%$ ethanol for $3 \mathrm{~min}$, 95\% ethanol for $3 \mathrm{~min}, 80 \%$ ethanol for $3 \mathrm{~min}$ and $70 \%$ ethanol for $3 \mathrm{~min}$ ) and washing in distilled water for $5 \mathrm{~min}$. Sections (thickness, $5 \mu \mathrm{m}$ ) were cut for hematoxylin-eosin staining and immunohistochemistry. Hematoxylin-eosin staining was performed at room temperature according to the following protocol: i) Staining in hematoxylin solution for 8 min; ii) washing with $\mathrm{H}_{2} \mathrm{O}$ for $5 \mathrm{~min}$; iii) differentiation in $1 \%$ acid ethanol for $30 \mathrm{sec}$; iv) washing with $\mathrm{H}_{2} \mathrm{O}$ for $1 \mathrm{~min}$; v) bluing in $0.2 \%$ ammonia water for $40 \mathrm{sec}$; vi) washing with $\mathrm{H}_{2} \mathrm{O}$ for 5 min; vii) counterstaining in $1 \%$ eosin solution for 5 min; viii) washing with $\mathrm{H}_{2} \mathrm{O}$ for 2 min; ix) dehydration in increasing concentrations of ethanol and clearing in xylene; and $\mathrm{x}$ ) mounting with xylene-based mounting medium. Immunohistochemical staining was performed using monoclonal antibodies (Sigma-Aldrich; Merck KGaA, Darmstadt, Germany) directed against myeloperoxidase (MPO; dilution, 1:1,000; cat. no., HPA021147), glial fibrillary acidic protein (GFAP; dilution, 1:1,000; cat. no., HPA056030), synaptophysin (SYN; dilution, 1:200; cat. no., SAB5500180), neurofilament protein (NF; dilution, 1:1,000; cat. no., SAB4700772), epithelial membrane antigen (EMA; dilution, 1:200; cat. no., HPA004179), leukocyte common antigen [LCA/cluster of differentiation (CD) 45; dilution, 1:50; cat. no., SAB1306481], lysozyme (dilution, 1:500; cat. no., HPA039179), CD3 (dilution, 1:150; cat. no., SAB5500057), CD10 (dilution, 1:100; cat. no., SAB5500035), CD20 (dilution, 1:100; cat. no., SAB5500049), CD33 (dilution, 1:200; cat. no.,HPA035832), CD34 (dilution, 1:200; cat. no., HPA036722), CD38 (dilution, 1:100; cat. no., SAB5500063), CD56 (dilution, 1:200; cat. no.,HPA039835), CD68 (dilution, 1:100; cat. no., SAB5500070), CD99 (dilution, 1:200; cat. no., HPA035304), CD117 (dilution, 1:200; cat. no., SAB4700750), S-100 calcium-binding protein (S-100; dilution, 1:100; cat. no., SAB4200671), and proliferation marker protein Ki-67 (Ki-67; dilution, 1:200; cat. no., SAB5500134) (12). The antibodies were diluted in $5 \%$ bovine serum albumin (Sigma-Aldrich; Merck KGaA), and the tissues were incubated overnight at $4^{\circ} \mathrm{C}$. Following use of the color developing reagent (3,3'-diaminobenzidine; Sigma-Aldrich; Merck KGaA) to reveal the staining, the slides were analyzed using an Olympus BX51 microscope equipped with an Olympus DP71 digital camera (Olympus Corporation, Tokyo, Japan).

The laboratory data were collected, including complete blood counts, serum biochemical parameters and hepatorenal functions. Magnetic resonance imaging (MRI) scans were performed using a 3.0T scanner (Signa Excite 3.0T; GE Healthcare Bio-Sciences, Pittsburgh, PA, USA), including routine T1-weighted (TE/TR=10-20/500-700 ms), T2-weighted (TE/TR=100-120/2700-3700 ms) and gadolinium-diethylenetriamine pentaacetic acid (Gd-DTPA) contrasted T1-weighted (TE/TR=10-20/400-800 ms) sequences. Additionally, diffusion-weighted imaging (TE/TR=90/6700 ms) was performed in 1 patient (case two); computed tomography (CT) scans were performed for 2 patients (cases two and four) using a 64-detector CT scanner (LightSpeed VCT, GE Healthcare Bio-Sciences). Follow-up data was collected for all patients. The clinical and radiological profiles of these patients are summarized in Table I.

\section{Case reports}

Case one. A 27-year-old male presented with a 5-month history of numbness and weakness in the lower extremities accompanied by sciatica. The baseline laboratory examinations of the peripheral blood and the bone marrow at admission were all normal. Neurological examination revealed a loss of sensation below the lumbar (L)1 dermatome and grade 1/5 bilateral weakness of the lower extremities. Spinal MRI scans detected multiple masses in the spinal canal in the thoracic (T)12-sacral (S)1 region and the sacral giant cell mass extended out from the spinal canal into the paraspinal region. The masses were isointense on the T1- and T2-weighted MRI scans. Following contrast agent administration, the masses demonstrated marked homogeneous enhancement (Fig. 1). Gross total resection of the masses in the T12-L3 region was performed, whereas the mass at the L5-S1 level was partially resected for decompression. Intraoperatively, the lesions were intradural and highly attached to the cauda equina. Immunohistological examination revealed the presence of myeloid sarcoma, with positive staining for MPO, LCA, lysozyme, and CD10, 56, 68, 99 and 117, but negative staining results for GFAP, SYN, NF, EMA, CD20 and S-100. The marker of proliferation Ki-67 labeling index (12) was $\sim 60 \%$. Repeated examinations of the peripheral blood and the bone marrow revealed no abnormalities. Considering the potential leukemic risk, an aggressive induction chemotherapy regime for leukemia was administered. The patient's postoperative course was without adverse events and the sciatica was completely relieved. During the follow-up period of 9 months, the patient's neurological functions improved.

Case two. A 27-year-old male presented with a 6-month history of right tinnitus and hearing loss. Three months prior to hospital admission, the patient developed right-sided facial pain. There were no relevant findings in the patient's prior medical history. Laboratory data were all within normal limits, and no hematological abnormalities were noted. A CT scan of the brain revealed a hyperdense mass in the right cerebellopontine angle. A further MRI scan of the brain revealed a dumbbell-shaped mass that appeared isointense on the T1- and T2-weighted images, with marked homogeneous enhancement. Diffusion-weighted imaging demonstrated restricted diffusion of the mass (Fig. 2). A presumptive diagnosis of schwannoma and secondary trigeminal neuralgia was made. Intraoperatively, the right trigeminal, oculomotor and trochlear nerves were observed to be enveloped and infiltrated by the intradural tumor. The tumor was subtotally resected in a piecemeal manner. Immunohistological examination revealed myeloid sarcoma, with positive staining for MPO, LCA, lysozyme, and CD10, 33, 38, 45, 56, 68, 99 and 117, but no staining for SYN, GFAP, NF, EMA, CD20 or S-100. The Ki-67 labeling index was $\sim 80 \%$. The patient's postoperative course was without adverse 


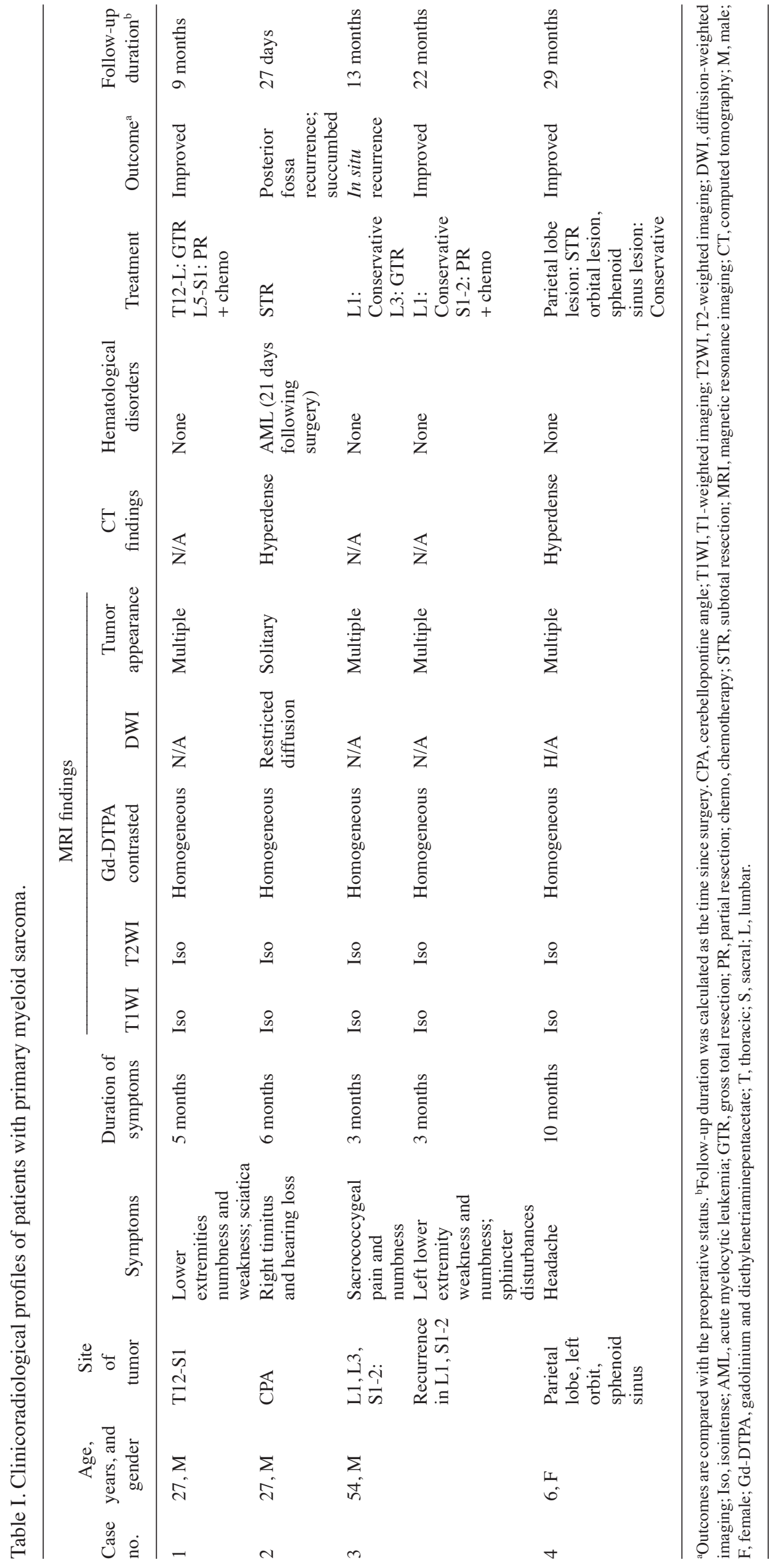



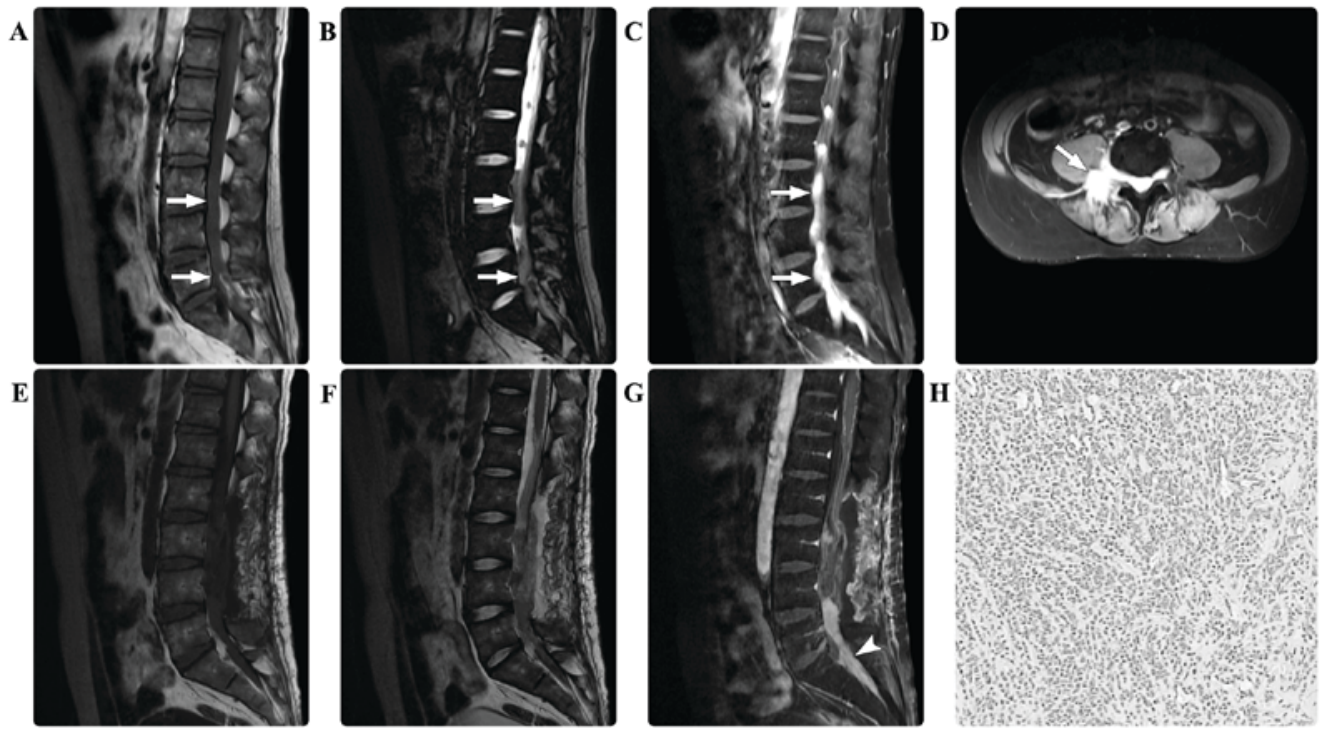

Figure 1. In case one, the preoperative spinal magnetic resonance imaging scan revealed multiple masses (arrows) in the T12-S1 region. The masses were isointense on (A) T1-weighted and (B) T2-weighted images. (C) Following contrast agent administration, the masses demonstrated marked homogeneous enhancement. (D) The axial enhanced image revealed that the mass extended from the spinal canal into the paraspinal region. The postoperative (E) T1-weighted, (F) T2-weighted and (G) contrasted T1-weighted images demonstrated that the masses in the T12-L3 regions had undergone effective gross total resection, and that the mass (arrowhead) at the L5-S1 level had been partially resected for decompression. Pathological hematoxylin and eosin staining of the resected tissue revealed myeloid sarcoma (magnification, 200x). T12, thoracic 12; S, sacral; L, lumbar.
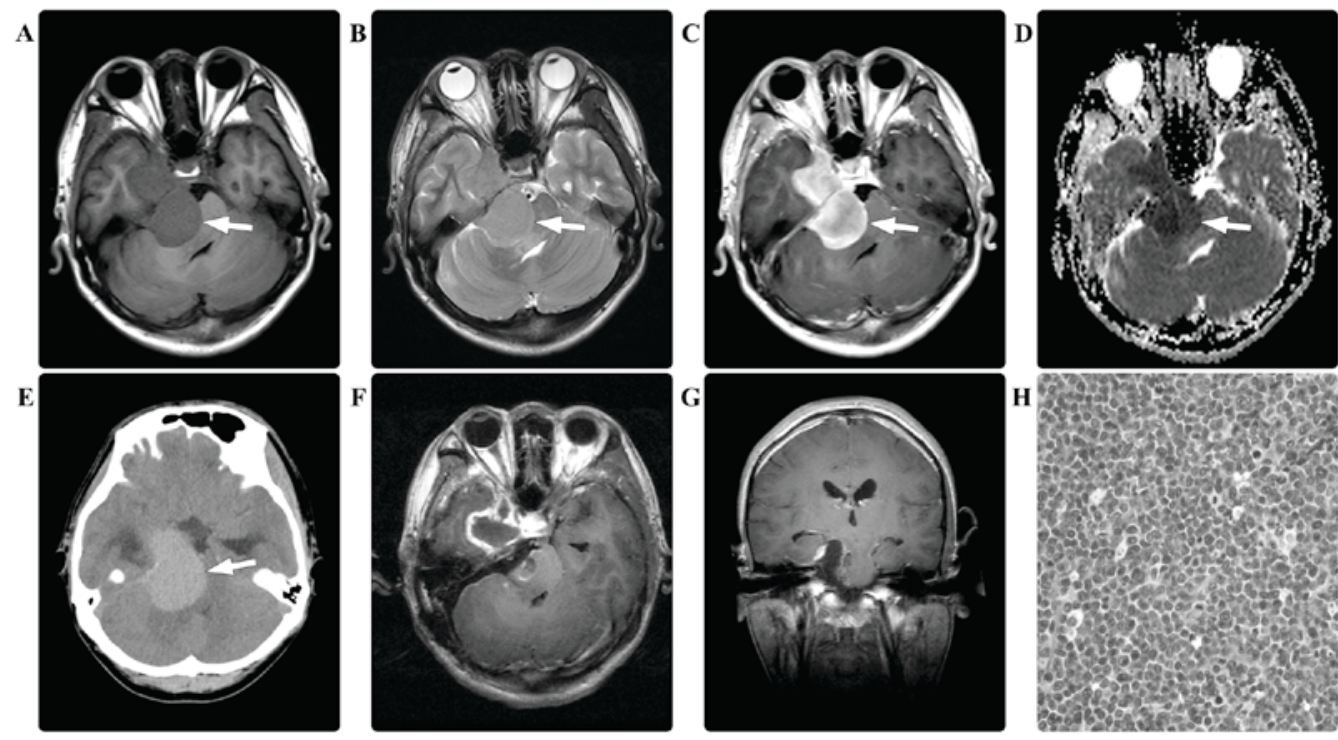

Figure 2. In case two, the preoperative brain magnetic resonance imaging scan revealed a dumbbell shaped mass (arrows) in the right cerebellopontine angle, which appeared isointense on (A) T1-weighted and (B) T2-weighted images, with (C) marked homogeneous enhancement. (D) The diffusion-weighted imaging demonstrated restricted diffusion (arrow). (E) The computed tomography scan of the brain revealed a hyperdense lesion (arrow). Postoperatively, the (F) enhanced axial and (G) coronal T1-weighted images demonstrated a subtotal resection of the tumor. Pathological hematoxylin and eosin staining of the resected tissue revealed myeloid sarcoma (magnification, $\mathrm{x} 400$ ).

events, laboratory indices were normal and he was discharged 1 week following surgery. At 3 weeks post-surgery, the patient was re-admitted due to vomiting, dysphagia and paraplegia. An MRI scan of the brain revealed multiple masses with an isointense appearance on T1- and T2-weighted images, and homogeneous enhancement in the posterior fossa, which was presumed to indicate the recurrence of MS. Hematological examinations revealed AML. Due to the disease progression and a poor Karnofsky performance scoring 20 points, no further surgical intervention was considered. Supportive treatment (including oxygen and assisted ventilation, monitoring, fluid and nutrition) and an AML-specific induction chemotherapy regimen (including idarubicin at $45 \mathrm{mg} / \mathrm{m}^{2} /$ day for 3 days and cytarabine at $100 \mathrm{mg} / \mathrm{m}^{2} /$ day for 5 days) were scheduled. However, the patient succumbed to the disease 6 days following his re-admission.

Case three. A 54-year-old male presented with a 3-month history of pain and numbness in the sacrococcygeal region. There were no relevant findings in the patient's prior medical 

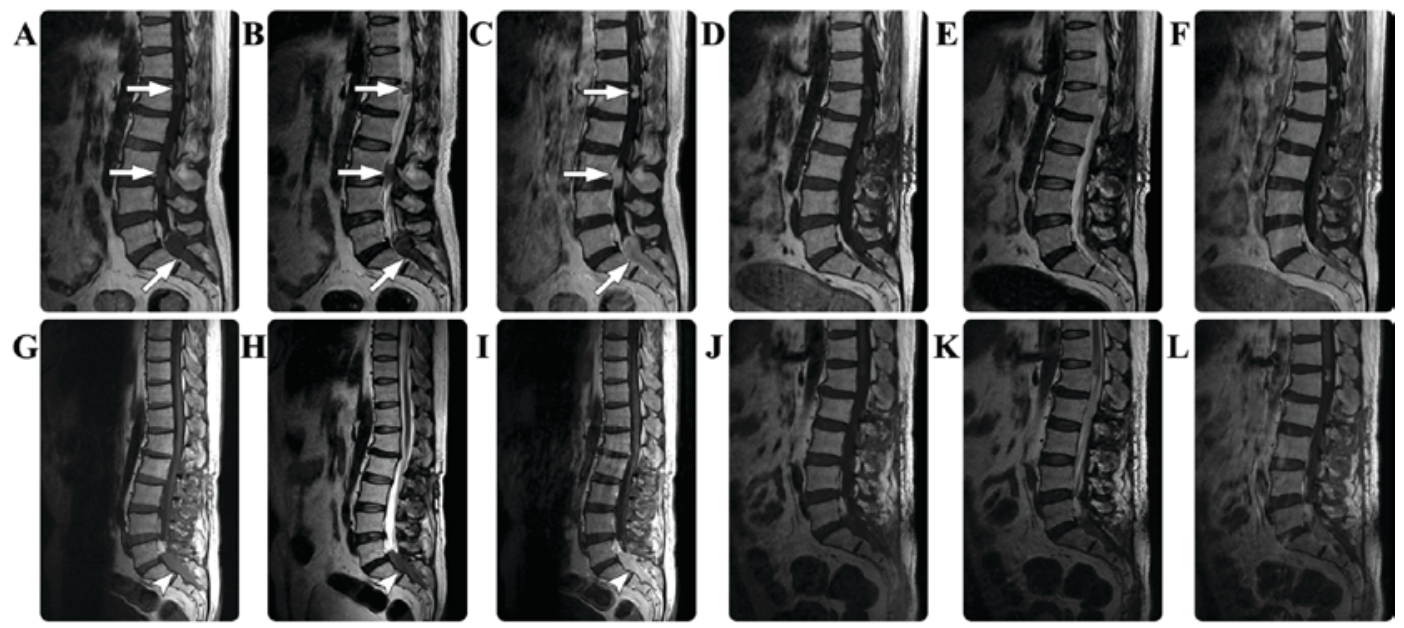

Figure 3. In case three, the preoperative spinal MRI scan revealed multiple masses (arrows) in the spinal canal in the L1, L3 and S1-S2 regions, which appeared isointense on the (A) T1-weighted and (B) T2-weighted images, with (C) marked homogeneous enhancement. The postoperative (D) T1-weighted, (E) T2-weighted and (F) contrasted T1-weighted images demonstrated that the mass at in the S1-S2 region had been partially resected. Twelve months following surgery, a repeated spinal MRI revealed in situ progression (arrowheads) of the sacral residual tumor in the (G) T1-weighted, (H) T2-weighted and (I) contrasted T1-weighted images. Subsequently, a second surgical resection of this tumor was performed, and postoperative MRI scans revealed residual tumor in the (J) T1-weighted, (K) T2-weighted and (L) contrasted T1-weighted images. MRI, magnetic resonance imaging; S, sacral; L, lumbar.

history. The results of routine laboratory examinations were all normal and no hematological abnormalities were noted. Physical examination detected no sensorimotor disturbances. An MRI scan of the spine revealed multiple masses in the spinal canal in the L1, L3 and S1-S2 regions, and a giant cell sacral mass extended from the spinal canal into the paraspinal region. The masses were isointense on T1- and T2-weighted images. Following contrast agent administration, the masses underwent marked homogeneous enhancement (Fig. 3). A diagnosis of multiple schwannomas was suspected. Gross total resection was performed on the tumor in the $\mathrm{L} 3$ region; however, the tumor at the S1-S2 levels was partially resected due to its diffuse paraspinal infiltration. Intraoperatively, the lesions were epidural and highly attached to the nerve roots. Immunohistological examination revealed myeloid sarcoma, with positive staining for MPO, LCA, lysozyme, and CD3, 10, 33, 34, 38, 45, 56, 68, 99 and 117, but negative staining results for SYN, GFAP, NF, EMA, CD20 and S-100. The Ki-67 labeling index was $20 \%$. The patient's postoperative course was without adverse events and standard laboratory test results were all normal. Twelve months following surgery, the patient was readmitted to hospital with a 3-month history of left lower extremity weakness and numbness, and sphincter disturbances. Physical examination revealed a loss of sensation below the $\mathrm{S} 1$ dermatome and grade $3 / 5$ decreased muscle strength in the left lower extremity. Repeated spinal MRI scans detected an in situ progression of the sacral residual tumor. A second surgical resection of this tumor was performed; however, a complete resection was unable to be achieved. A comprehensive hematological evaluation, including cytological examinations and chromosome analysis, was performed, but no abnormalities were identified. Due to the potential presence of a myeloblastic disorder and the poor prognosis, an anti-leukemia chemotherapy regimen composed of dasatinib (100 mg/day) and Gleevec (400 mg/day) was recommended. The patient underwent this chemotherapy for 3 consecutive months and subsequently stopped due to financial reasons. During the follow-up period, the patient's neurological functions improved gradually. At a total of 22 months following the second surgery, the patient was neurologically asymptomatic.

Case four. A 6-year-old female presented with a recurrent headache for 10 days. There were no relevant findings in the patient's prior medical history. The baseline laboratory examination results were all normal. A CT scan of the brain revealed multiple intracranial hyperdense masses. An MRI scan of the brain subsequently identified a mass in the parietal lobe, the left orbit and the sphenoid sinus. The masses were all isointense on the T1- and T2-weighted images, with marked homogeneous enhancement (Fig. 4). A diagnosis of multiple schwannoma or Rosai-Dorfman disease was suspected. Due to the symptom of left frontoparietal headache, a surgical resection of the parietal tumor was scheduled. Intraoperatively, the dura mater and local skull were observed to be infiltrated by the tumor. Subtotal resection of the tumor was performed in a piecemeal manner and the peripherally infiltrated dura mater was fulgurated. Immunohistological examination revealed myeloid sarcoma, with positive staining for MPO, LCA, lysozyme, and CD10, 33, 34, 45, 68, 99 and 117, but no staining for SYN, GFAP, NF, EMA, CD20, vimentin or S-100. The Ki-67 labeling index was $\sim 16 \%$. The patient's postoperative course was without adverse events and the headache was relieved. The results of repeated laboratory examinations were all normal. No adjuvant radiotherapy or chemotherapy was performed due to the parents' refusal. During the follow-up period of 29 months, no disease progression was detected.

\section{Discussion}

MS has previously been termed chloroma, extramedullary myeloid tumor or granulocytic sarcoma (13). The term chloroma is derived from the Greek word chloros (green), as the appearance of the lesion is typically green due to high levels 

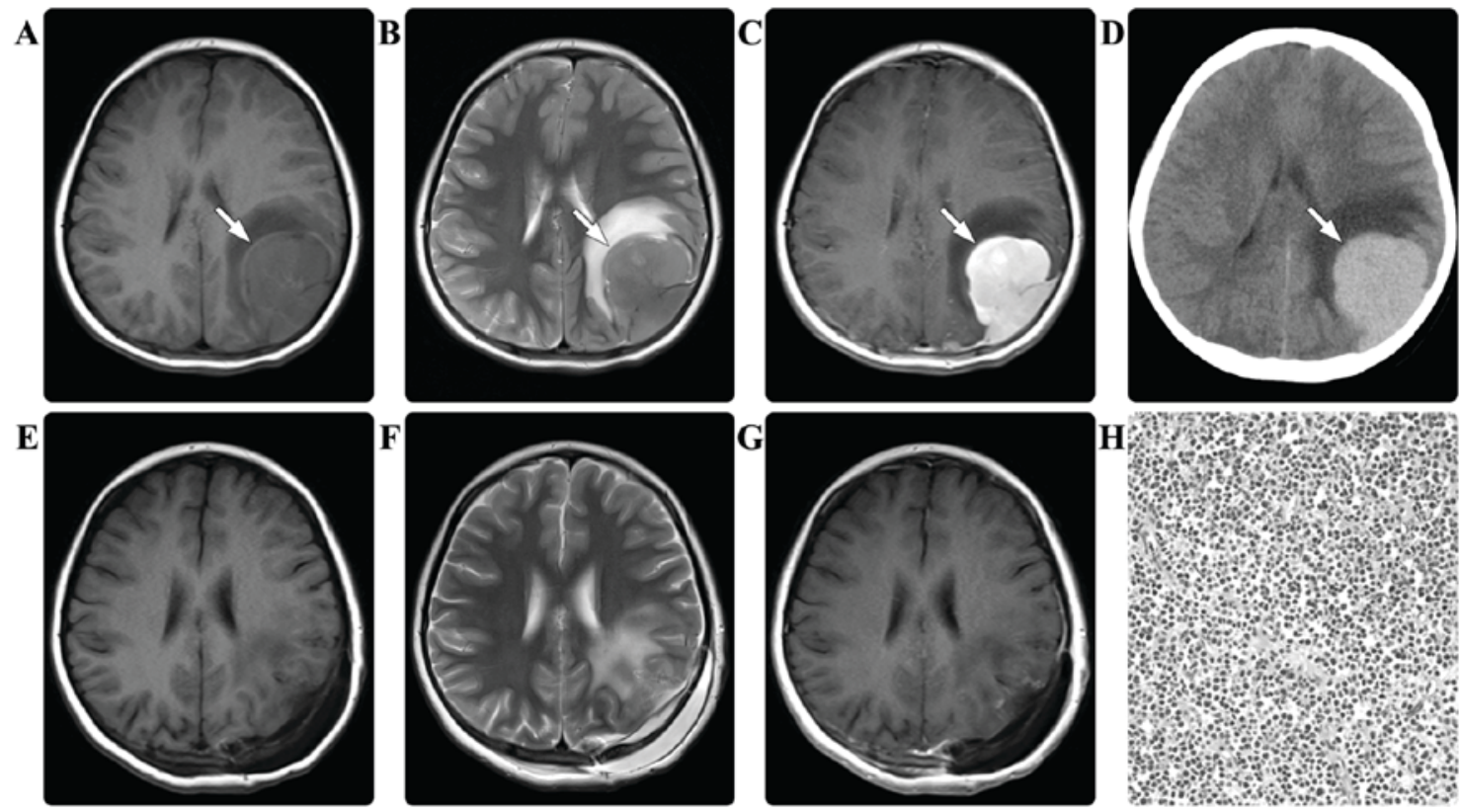

Figure 4. In case four, the magnetic resonance imaging scan of the brain revealed a mass (arrows) in the left parietal lobe, which appeared isointense on the (A) T1-weighted and (B) T2-weighted images, with (C) marked homogeneous enhancement. (D) The computed tomography scan revealed that the mass (arrow) was hyperdense. The postoperative (E) T1-weighted, (F) T2-weighted and (G) contrasted T1-weighted images demonstrated that the mass had been subtotally resected. Pathological hematoxylin and eosin staining of the resected tissue revealed myeloid sarcoma (magnification, x200).

of myeloperoxidase expression; however, this is not always applicable as the color may rapidly fade upon oxygen exposure (14). The 2008 World Health Organization classification adopted the term 'myeloid sarcomas' as a subgroup of 'acute myeloid leukemias, not otherwise categorized' (15). This type of malignancy frequently occurs during the course of active hematological disease, including acute or chronic leukemia and myeloproliferative disorder, or occurs during remission from leukemia (1-4). CNS-MS is uncommon, and it has been suggested that it may occur when leukemic cells pass from the bone marrow of the skull to the dura, subarachnoid space and Virchow-Robin spaces, resulting in invasion of the brain surface (16). However, there remains a highly rare variant, primary CNS-MS, presenting as isolated solid neoplasms in the brain or spinal cord and occurring de novo with no apparent signs or symptoms of concomitant hematological disease $(5,6,11)$.

According to the classification proposed by Audouin et al (17), there are four varied patterns of MS: i) MS that develops during the active phase of leukemia; ii) MS that develops alongside known chronic myeloproliferative disorders; iii) MS that manifests as a relapse months or years following clinical remission from AML, particularly following bone marrow transplantation; and iv) MS preceding AML diagnosis, which may be detected in previously healthy individuals with no blast cell infiltration into the bone marrow and a normal peripheral blood cell count. As described by Krause (18), patients with MS without leukemic evidence accounted for $0.6 \%$ of all MS cases. The pathogenesis of isolated CNS-MS without bone marrow involvement remains to be elucidated; however, relevant clinical profiles suggest that CNS-MS may portend a potential or delayed leukemia (19-21). According to a previous study, the majority of patients with isolated CNS-MS may progress to myeloid leukemia with blast cell infiltration at an average of 10.5 months following the pathological diagnosis of MS (3).

Clinical manifestations of CNS-MS are nonspecific and are associated with increased intracranial pressure or space-occupying effects $(6,11)$. The radiological features of CNS-MS are typically characterized by the presence of an isodense or hyperdense mass on CT scan images, with marked homogeneous enhancement following contrast agent administration (22). The appearance of CNS-MS via MRI is typically hypointense to isointense on T1- and T2-weighted images, with marked enhancement following the administration of a contrast agent $(23,24)$. Radiological differential diagnoses may include meningioma, lymphoma and schwannoma. Obtaining a preoperative diagnosis is challenging and isointense signal characteristics may be suggestive of CNS-MS.

The definitive diagnosis of CNS-MS may depend upon pathological and immunohistochemical evidence. MPO is a specific antigen marker for MS (25). In the present study, the tumor specimens from all patients were positive for MPO, CD68 and CD45, which is consistent with data from a previous study (26). Histological differential diagnoses comprise various small cell tumors, including Burkitt's lymphoma, embryonal rhabdomyosarcoma and Ewing's sarcoma.

The optimal treatment for CNS-MS remains the subject of debate. The typical therapeutic option is a combination of local surgical resection and chemotherapy $(1-4,11,26)$. However, there are several challenges to treatment that arise as a result of isolated CNS-MS. Complete surgical resection is challenging due to the extensive infiltration of MS into the surrounding tissues and, in the majority of cases, only subtotal or partial resection is achieved. In the present study, an in situ progression of the residual tumor was observed in case three. In this case, complete surgical resection was difficult and not enforced in the second surgical procedure, with 
adjuvant chemotherapy aiding long-term tumor remission. Additionally, the administration of chemotherapy remains problematic for cases of primary CNS-MS without leukemic evidence. In the present study, case one was managed using an aggressive induction chemotherapy regime for leukemia; the clinical improvement and event-free follow-up in this patient supports the decision to perform an aggressive treatment. In case two, chemotherapy was not administrated in a timely manner and upon the detection of leukemia the condition was not manageable. In case four, 1 tumor was subtotally resected and the remaining 2 tumors were conservatively observed. Although no chemotherapy was administered, the disease did not progress during the 29-month follow-up period. The low Ki-67 index may provide an explanation for the indolent disease course in this patient. Considering the risk of progression to leukemia, the results of the present study indicate that systematic chemotherapy be included in the treatment for all CNS-MS cases.

In conclusion, primary CNS-MS without leukemic evidence is highly rare and has distinctive radiological characteristics. Due to its diffuse tissue infiltration, completely surgical resection of this type of tumor is challenging. It is important for clinicians to be aware of potential hematological disorders in patients with CNS-MS. A combined surgical and chemotherapeutic strategy may facilitate long-term remission in this group.

\section{References}

1. Collins $\mathrm{C}$ and Knoderer H: Central nervous system involvement at the time of presentation in acute promyelocytic leukemia. Pediatr Blood Cancer 54: 603-605, 2010.

2. Colović N, Colović M, Cemerikić V, Terzić T, Ivanović S, Skender M and Bosković D: Granulocytic sarcoma of the brain in a patient with acute myeloid leukemia. Acta Chir Iugosl 51: 129-131, 2004.

3. Cervantes GM and Cayci Z: Intracranial CNS manifestations of myeloid sarcoma in patients with acute myeloid leukemia: Review of the literature and three case reports from the author's institution. J Clin Med 4: 1102-1112, 2015.

4. Cho SF, Liu TC and Chang CS: Isolated central nervous system relapse presenting as myeloid sarcoma of acute myeloid leukemia after allogeneic peripheral blood stem cell transplantation. Ann Hematol 92: 133-135, 2013.

5. Piñán MA, Ardanaz MT, Guinea JM and García-Ruiz JC: Myeloid sarcoma preceding an acute promyelocytic leukaemia with neuromeningeal infiltration. Ann Hematol 93: 339-340, 2014

6. Widhalm G, Dietrich W, Müllauer L, Streubel B, Rabitsch W, Kotter MR, Knosp E and Roessler K: Myeloid sarcoma with multiple lesions of the central nervous system in a patient without leukemia. Case report. J Neurosurg 105: 916-919, 2006.

7. Breccia M, D'Andrea M, Mengarelli A, Morano SG, D'Elia GM and Alimena G: Granulocytic sarcoma of the pancreas successfully treated with intensive chemotherapy and stem cell transplantation. Eur J Haematol 70: 190-192, 2003.
8. Fitoz S, Atasoy C, Yavuz K, Gozdasoglu S, Erden I and Akyar S: Granulocytic sarcoma. Cranial and breast involvement. Clin Imaging 26: 166-169, 2002.

9. Aki H, Baslar Z, Uygun N, Ozguroglu M and Tuzuner N: Primary granulocytic sarcoma of the urinary bladder: Case report and review of the literature. Urology 60: 345, 2002.

10. Pileri SA, Ascani S, Cox MC, Campidelli C, Bacci F, Piccioli M, Piccaluga PP, Agostinelli C, Asioli S, Novero D, et al: Myeloid sarcoma: Clinico-pathologic, phenotypic and cytogenetic analysis of 92 adult patients. Leukemia 21: 340-350, 2007.

11. Yang C, Liu Y, Li G, Bai J, Qian J and Xu Y: Multifocal myeloid sarcoma in the central nervous system without leukemia. Clin Neurol Neurosurg 120: 99-102, 2014.

12. Karamitopoulou E, Perentes E, Diamantis I and Maraziotis T: Ki-67 immunoreactivity in human central nervous system tumors: A study with MIB 1 monoclonal antibody on archival material. Acta Neuropathol 87: 47-54, 1994.

13. Campidelli C, Agostinelli C, Stitson R and Pileri SA: Myeloid sarcoma: Extramedullary manifestation of myeloid disorders. Am J Clin Pathol 132: 426-437, 2009.

14. Cho WH, Choi YJ, Choi BK and Cha SH: Isolated recurrence of intracranial granulocytic sarcoma mimicking a falx meningioma in acute myeloblastic leukemia. J Korean Neurosurg Soc 47: 385-388, 2010.

15. Vardiman JW, Thiele J, Arber DA, Brunning RD, Borowitz MJ, Porwit A, Harris NL, Le Beau MM, Hellström-Lindberg E, Tefferi A and Bloomfield CD: The 2008 revision of the World Health Organization (WHO) classification of myeloid neoplasms and acute leukemia: Rationale and important changes. Blood 114: 937-951, 2009.

16. Azzarelli V and Roessmann U: Pathogenesis of central nervous system infiltration in acute leukemia. Arch Pathol Lab Med 101: 203-205, 1977.

17. Audouin J, Comperat E, Le Tourneau A, Camilleri-Broët S, Adida C, Molina T and Diebold J: Myeloid sarcoma: Clinical and morphologic criteria useful for diagnosis. Int J Surg Pathol 11: 271-282, 2003.

18. Krause JR: Granulocytic sarcoma preceding acute leukemia: A report of six cases. Cancer 44: 1017-1021, 1979.

19. Hurwitz BS, Sutherland JC and Walker MD: Central nervous system chloromas preceding acute leukemia by one year. Neurology 20: 771-775, 1970.

20. Llena JF, Kawamoto K, Hirano A and Feiring EH: Granulocytic sarcoma of the central nervous system: Inital presentation of leukemia. Acta Neuropathol 42: 145-147, 1978.

21. Piñán MA, Ardanaz MT, Guinea JM and García-Ruiz JC: Myeloid sarcoma preceding an acute promyelocytic leukaemia with neuromeningeal infiltration. Annals Hematol 93: 339-340, 2014.

22. Sowers JJ, Moody DM, Naidich TP, Ball MR, Laster DW and Leeds NE: Radiographic features of granulocytic sarcoma (chloroma). J Comput Assist Tomogr 3: 226-233, 1979.

23. Chaudhry AA, Gul M, Chaudhry AA and Dunkin J: Qualitative assessment of diffusion weighted imaging and susceptibility weighted imaging of myeloid sarcoma involving the brain. J Comput Assist Tomogr 40: 61-66, 2016.

24. Pui MH, Fletcher BD and Langston JW: Granulocytic sarcoma in childhood leukemia: Imaging features. Radiology 190: 698-702, 1994.

25. Pinkus GS and Pinkus JL: Myeloperoxidase: A specific marker for myeloid cells in paraffin sections. Mod Pathol 4: 733-741, 1991.

26. Qian J, Cui QU, Liu Y, Li X, Sun X, Zhu H and Wang C: Isolated primary intracranial myeloid sarcoma with neuromeningeal infiltration: A case report. Oncol Lett 9: 1647-1650, 2015. 